\title{
Biomicroscopy of papillae associated with wearing of soft contact lenses
}

\author{
DONALD R. KORB, ${ }^{1}$ JACK V. GREINER, ${ }^{2}$ VICTOR M. FINNEMORE, 1 \\ AND MATHEA R. ALLANSMITH ${ }^{3}$ \\ From ' 80 Boylston Street, Boston, Massachusetts; the Departments of Ophthalmology, \\ ${ }^{2}$ University of Illinois Eye and Ear Infirmary, Chicago, and ${ }^{3}$ Harvard Medical School, Boston; \\ the ${ }^{23}$ Department of Cornea Research, Eye Research Institute of Retina Foundation, Boston; and the \\ ${ }^{2}$ Department of Pathology, Chicago College of Osteopathic Medicine, Chicago, USA
}

SUMMARY We studied the topographical, macrostructural, and fluorescein staining characteristics of papillary changes of the upper tarsal conjunctiva associated with the wearing of hydrophilic (soft) contact lenses. Fifty soft contact lens wearers with elevated conjunctival papillae $>0.3 \mathrm{~mm}$ in diameter were studied. Topographic characteristics recorded were distribution and number of papillae; macrostructural characteristics recorded were diameter and morphology of papillae. The information collected included age of patient, duration of lens wear, average daily time of wearing lens, presence or absence of itching or mucus, refractive status, and atopic history. Papillae were found in most cases in the conjunctival zone adjacent to the tarsal fold and were never found in the zone adjacent to the eyelid margin without also occurring in the intervening zone. The diameter of the papillae ranged from $>0.3 \mathrm{~mm}$ to $2.0 \mathrm{~mm}$. The number of papillae per eyelid ranged from 4 to over 100 . The apices of the papillae were frequently flattened, and these flattened surfaces frequently stained with fluorescein. The vascular supply of individual papillae was observed to radiate from a vessel occupying the central core of each papilla.

Clinically observable changes in the upper tarsal conjunctiva have been reported in the wearers of both hard and soft contact lenses. ${ }^{12}$ The macrostructural characteristics of the papillary changes of the upper tarsal conjunctiva associated with the wearing of polymethylmethacrylate (PMMA) contact lenses have been established. ${ }^{34}$ We questioned whether there are biomicroscopically observable characteristics that would permit differentiation between hard and soft contact lens papillary conjunctivitis based on the distribution, morphology, and staining characteristics of papillae, and the degree of hyperaemia.

The purpose of the present study was to determine the characteristics of changes of the upper tarsal conjunctiva associated with the wearing of soft contact lenses, and to compare and contrast these characteristics with those changes associated with the wearing of hard contact lenses. ${ }^{4}$

Correspondence to Mathea R. Allansmith, MD, 20 Staniford St, Boston, MA 02114, USA.

\section{Subjects and methods}

Subject selection. Fifty subjects with elevated conjunctival papillae $>0.3 \mathrm{~mm}$ in diameter associated with hydrophilic (soft) contact lens wearing were selected for study. Since standardised eyelid examinations had not been performed at regular intervals on all subjects, neither the onset nor the duration of this condition could be determined. Each subject's age, duration of lens wear, average daily lens wearing time, symptoms of itching and/or mucus, refractive status, and atopic history were obtained. Subjects wore their contact lenses for at least 2 hours before examination. Since we observed that subjects who had not worn their lenses for several days presented a marked decrease in conjinctival hyperaemia and the number of fluorescein-stained papillae, we selected only those subjects who were able to wear lenses a minimum of 4 hours.

Method of examination. The upper eyelid was everted, and the tarsal conjunctival surface was examined with the biomicroscope under white light 


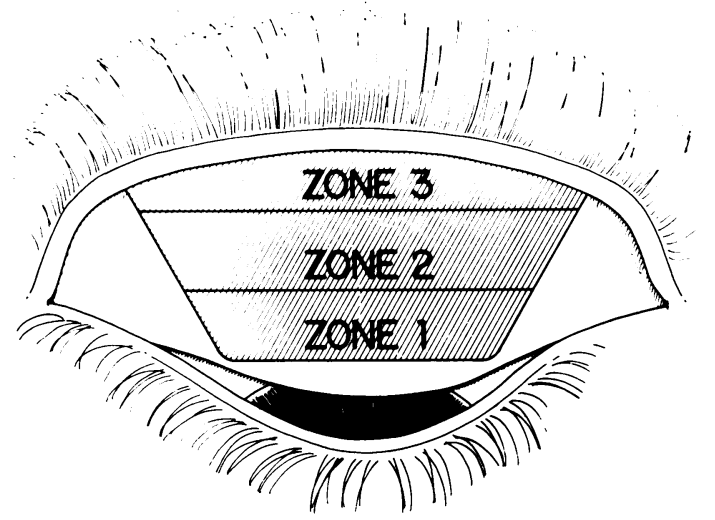

Fig. 1 Diagram illustrating the three zones of the tarsal conjunctiva (3).

and $16 \times$ magnification. The lid was returned to the normal position and 2 drops of sterile $2 \%$ sodium fluorescein were instilled into the lower cul-de-sac. Subjects were instructed to blink completely 6 times to distribute the fluorescein over the tarsal conjunctival surface. The upper eyelid was then re-everted, and biomicroscopic examination was repeated with cobalt blue light.

Papillae were considered elevated only when they were observed, with a slit-beam and $10 \times$ magnification, to protrude from the surrounding conjunctiva surface. The distribution, number, diameter, morphology, presence of absence of apical staining, and the vascular pattern of the elevated papillae were noted. To describe the distribution of papillae the conjunctiva over the tarsal plate was divided into 3 zones of equal width (Fig. 1). Papillary diameter was measured with the micrometer on the slit apparatus of the Haag-Streit 900 slit-lamp. The upper tarsal conjunctiva was photographed first with white light and then with cobalt blue light after fluorescein instillation. Papillae or follicles occurring on the junctional conjunctiva (Fig. 1) were not considered to be abnormal, as they are frequently present in healthy persons.

Hyperaemia of the upper tarsal conjunctiva was graded on a scale of zero to +3: zero represented the absence of hyperaemia; grades 1,2 , and 3 the categories of mild, moderate, and severe respectively.

\section{Results}

Fifty subjects with bilateral papillary changes (a total of 100 eyelids) were studied. The severity of papillary changes ranged from mild without symptoms to severe with almost total loss of wearing tolerance. The elevated papillae were usually discrete; their apices were frequently flattened (Fig. 2). Fluorescein staining of these flattened apices was observed in $\mathbf{3 0}$ of the 100 eyelids (Fig. 2).

Papillae occurred in zone 1 only in 73 eyelids, in zones 1 and 2 in 25 eyelids, and in zones, 1, 2, and 3 in 2 eyelids, but did not occur in zones 2 only or 3 only. The clinical appearance of this zonal distribution is illustrated in Fig. 3.

The number of papillae $>0.3 \mathrm{~mm}$ in diameter per eyelid ranged from 4 to over 100 (Fig. 4). Twenty-five eyelids had between 4 and 10 papillae, 22 had 11 to 20 papillae, 26 had 21 to 40 papillae, and 24 had more than 40 papillae. Papillae in 28 of 100 eyelids had evidence of staining.

The papillae ranged from $>0.3 \mathrm{~mm}$ to $2.4 \mathrm{~mm}$ in diameter (Fig. 5); various sizes were present in each eyelid. The largest papilla in 23 eyelids was $>0.3 \mathrm{~mm}$ to $<0.5 \mathrm{~mm}$; from $0.5 \mathrm{~mm}$ to $<1.0 \mathrm{~mm}$ in 69 eyelids; and $1.0 \mathrm{~mm}$ or greater in 8 eyelids.

Grade 1 conjunctival hyperaemia was present in 34 eyelids, grade 2 hyperaemia in 41 , and grade 3 hyperaemia in 18 (Fig. 6). Seven eyelids were not hyperaemic. Papillae were often vascularised by the centrifugal branching of a central vessel.

The distributions of the patients' age, duration of lens wear, refractive status, and average daily lens wearing time are presented in Figs. 7-10. Twelve of the 50 subjects were atopic. Symptoms of mucus or itching or both were reported by 36 of the 50 subjects. Forty-two subjects reported a decrease of tolerance to contact lens wearing associated with a foreignbody sensation and the presence of mucus and itching. In a previous study we stated that decreased lens tolerance, mucus, and itching were common but not universal in wearers of soft lenses. This finding is confirmed in the present study.

\section{Discussion}

In those cases in which papillae were limited to a singie zone this zone was always zone 1 . Papillae did not occur exclusively in either zone 2 or zone 3 . Papillae were not present simultaneously in zones 1 and 3 without also occurring in zone 2 . These findings suggest that papillae develop first in zone 1 ; zones 2 and 3 apparently become involved only after papillae develop in zone 1 . In contrast, papillae in wearers of hard PMMA contact lenses were never observed solely in zone 1 but did occur alone in zones 2 or $3 .^{3}$ This distributional difference between soft and hard lens papillary conjunctivitis is most likely due to the variations in lens materials (polymer), surface characteristics, geometries, and fitting characteristics between the smaller rigid PMMA lenses (average $8 \cdot 0-9.5 \mathrm{~mm}$ ) and the larger soft hydrophilic lenses (average 13.0-15.0 $\mathrm{mm}$ ).

Detection of papillae, particularly those $<0.50 \mathrm{~mm}$ 

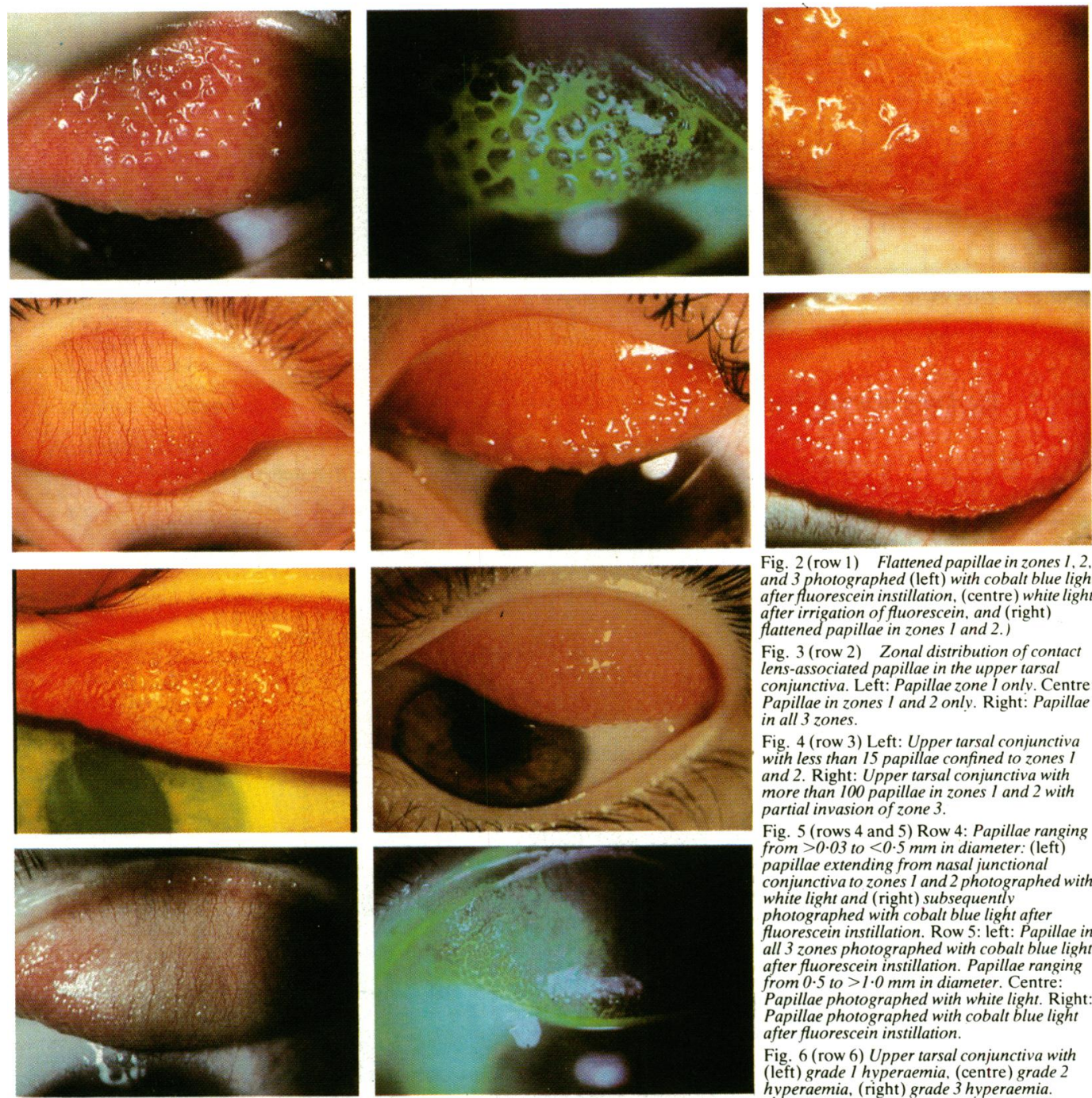

Fig. 2 (row 1) Flattened papillae in zones $I, 2$ and 3 photographed (left) with cobalt blue light after fluorescein instillation. (centre) white light after irrigation of fluorescein, and (right) flattened papillae in zones $I$ and 2.)

Fig. 3 (row 2) Zonal distribution of contact lens-associated papillae in the upper tarsal conjunctiva. Left: Papillae zone I onlv. Centre: Papillae in zones 1 and 2 only. Right: Papillae in all 3 zones.

Fig. 4 (row 3) Left: Upper tarsal conjunctiva with less than 15 papillae confined to zones and 2. Right: Upper tarsal conjunctiva with more than 100 papillae in zones 1 and 2 with partial invasion of zone 3 .

Fig. 5 (rows 4 and 5) Row 4: Papillae ranging from $>0.03$ to $<0.5 \mathrm{~mm}$ in diameter: (left) from $>0.03$ to $<0.5 \mathrm{~mm}$ in diameter: (left)
papillae extending from nasal junctional papillae extending from nasal junctional conjunctiva to zones 1 and 2 photograph
white light and (right) subsequently white light and (right) subsequently
photographed with cobalt blue light after fluorescein instillation. Row 5: left: Papillae in all 3 zones photographed with cobalt blue ligh after fluorescein instillation. Papillae ranging from 0.5 to $>1.0 \mathrm{~mm}$ in diameter. Centre: Papillae photographed with white light Right: Papillae photographed with cobalt blue light after fluorescein instillation.

Fig. 6 (row 6) Upper tarsal conjunctiva with (left) grade 1 hyperaemia, (centre) grade 2
hyperaemia, (right) grade 3 hyperaemia.
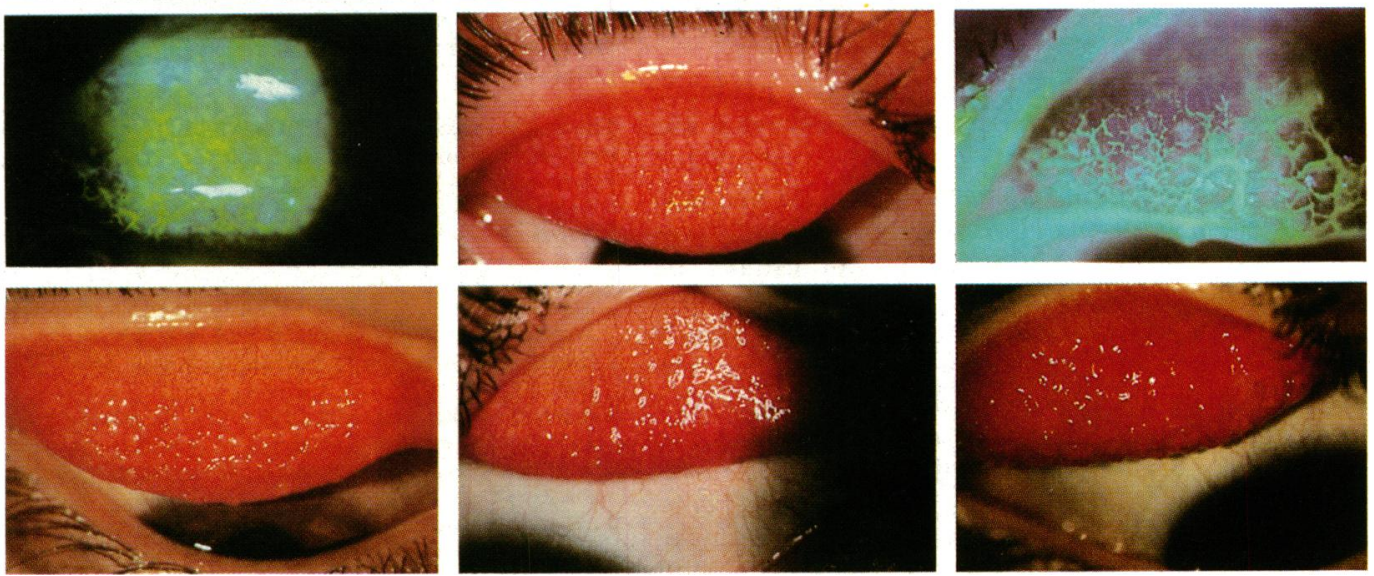


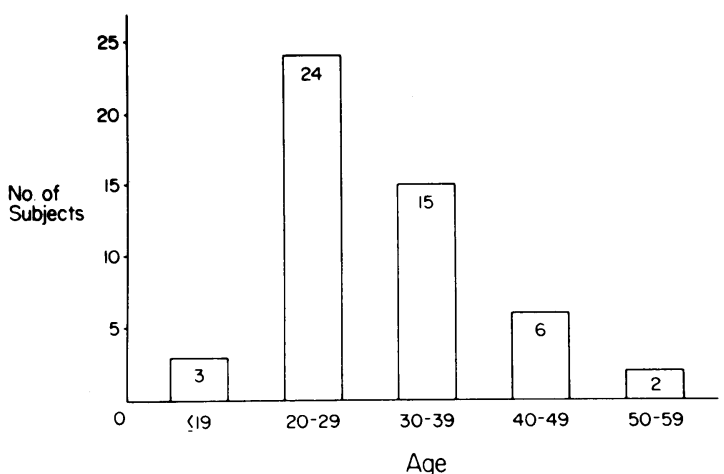

Fig. 7 Age distribution of subjects.

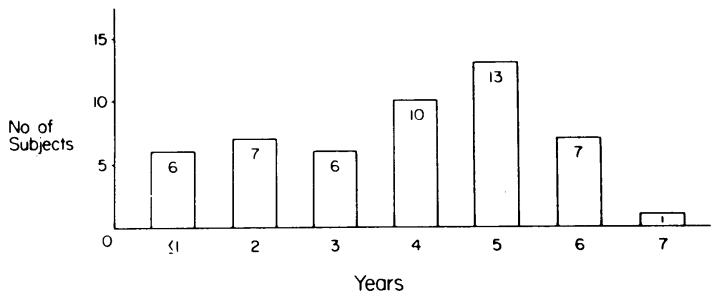

Fig. 8 Average duration of lens wear.

in diameter, frequently required cobalt blue light biomicroscopic examination of the tarsal conjunctiva after fluorescein staining. Papillae were elevated and usually round. The apices of the larger and more elevated papillae were frequently flattened and stained with fluorescein; papillae with the crater-like apical depressions characteristic of wearers of hard lenses were only rarely observed. ${ }^{3}$ We believe that the apical flattening is related to prolonged trauma to the upper tarsal conjunctiva. Since fluorescein staining occurs with epithelial cell damage, staining could be expected to be most severe at the apices of papillae. Eyelids of soft-lens wearers had a greater number of papillae $(>0.3 \mathrm{~mm}$ in diameter) than eyelids of hard-lens wearers. ${ }^{4}$

It is clear from the biomicroscopic evidence presented here and in our previous study ${ }^{4}$ that the clinical appearance of contact-lens-associated papillary conjunctivitis is characteristic for the type of contact lens worn. Hard and soft contact-lensassociated papillary conjunctivitis can usually be differentiated by topographical and macrostructural characteristics. However, in the case of soft-contact lens wearers with papillary conjunctivitis the symptom of itching, decreased tolerance to wearing a contact lens, and the sign of increased mucus are more severe than in wearers of hard contact lenses with papillary conjunctivitis. Interestingly, conjunctival hyperaemia was markedly increased in wearers of soft contact lenses in comparison with

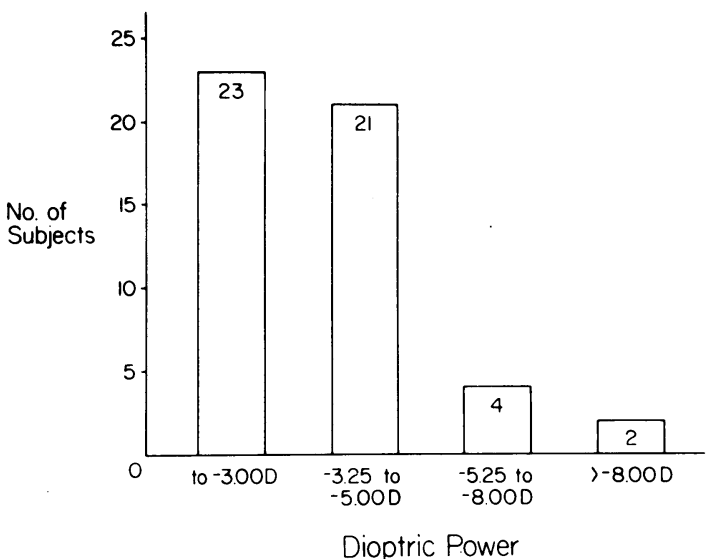

Fig. 9 Refractive status of subjects.

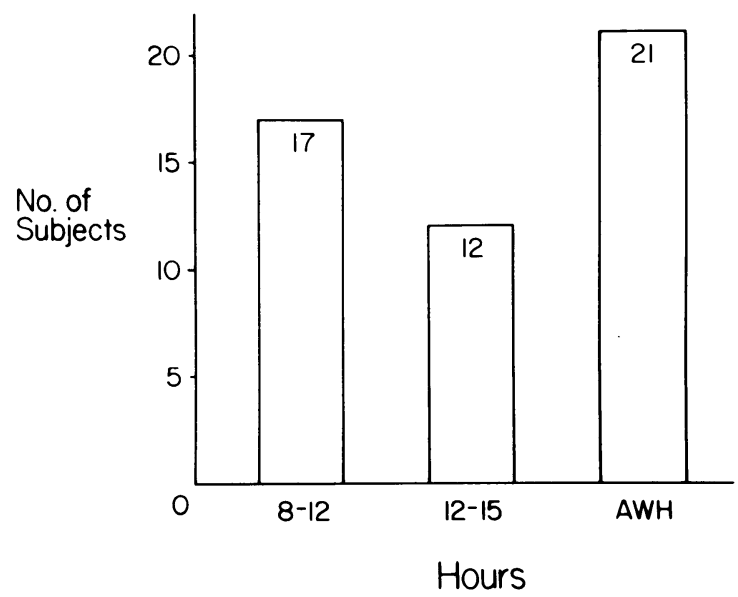

Fig. 10 Average daily lens wearing time.

wearers of hard lenses. ${ }^{4}$ This increase in hyperaemia may correlate with the increase in symptoms.

The authors thank Ms Donna Peace for technical assistance, Ms Ruth Zelkha for photographic reproductions, and Mr Robert Kelley for illustrations.

This study was supported by grant EY-02099 from the National Eye Institute, National Institutes of Health and by a research grant from Allergan, Inc.

\section{References}

1 Allansmith MR, Korb DR, Greiner JV, Henriquez AS, Simon MA, Finnemore VM. Giant papillary conjunctivitis in contact lens wearers. Am J Ophthalmol 1977; 83: 697-708.

2 Spring TG. Reaction to hydrophilic lenses. Med J Aust 1974; i: 499-500.

3 Korb DR, Allansmith MR, Greiner JV, Henriquez AS, Richmond PP, Finnemore VM. Prevalence of papillary changes associated with the wearing of hard contact lenses. Am J Ophthalmol 1980; 90: $336-41$.

4 Korb DR, Allansmith MR, Greiner JV, et al. Biomicroscopy of papillae associated with hard contact lens wearing. Ophthalmology 1981; 88: 1132-6. 